\title{
O Impacto da metodologia Lean para melhorar a segurança do paciente em hospitais
}

\author{
The Impact of Lean methodology to improve patient safety in hospitals \\ El impacto de la metodología Lean para mejorar la seguridad del paciente en los hospitales
}

Recebido: 03/10/2021 | Revisado: 09/10/2021 | Aceito: 15/10/2021 | Publicado: 18/10/2021

Francisca das Chagas Sheyla Almeida Gomes Braga

ORCID: https://orcid.org/0000-0001-5646-0100

Universidade Federal do Piauí, Brasil

E-mail: sheylagomesbraga@gmail.com

Daniel de Macêdo Rocha

ORCID: https://orcid.org/0000-0003-1709-2143

Universidade Federal do Piauí, Brasil

E-mail: daniel_m.rocha@outlook.com.br

Cléciton Braga Tavares

ORCID: https://orcid.org/0000-0003-4816-0858

Hospital de Urgência de Teresina, Brasil

Hospital Getúlio Vargas, Brasil

E-mail: bragatavares@yahoo.com.br

Geisa Machado Fontenelle

ORCID: https://orcid.org/0000-0002-7025-9195 Universidade Federal do Piauí, Brasil

E-mail: geysaenf@hotmail.com

Roxana Mesquita de Oliveira Teixeira Siqueira ORCID: https://orcid.org/0000-0001-9549-2068 Universidade Federal do Piauí, Brasil

E-mail: roxanasiqueira@hotmail.com

Antônio Francisco Machado Pereira ORCID: https://orcid.org/0000-0001-6682-1774 Universidade Federal do Piauí, Brasil

E-mail: Machado@ufpi.edu.br

Yara Maria Rêgo Leite

ORCID: https://orcid.org/0000-0002-4868-2624 Universidade Federal do Piauí, Brasil

E-mail: yara.leite@ebserh.gov.br

Veronica Elis de Araújo Rezende

ORCID: https://orcid.org/0000-0001-9076-3375

Universidade Federal do Piauí, Brasil

E-mail: veronicaelisrezende@yahoo.com.br

Adriana Jorge Brandão

ORCID: https://orcid.org/0000-0002-5539-0152

Universidade Federal do Piauí, Brasil E-mail: drithe@hotmail.com

Maria Lailda de Assis Santos

ORCID: https://orcid.org/0000-0002-5521-5151

Universidade Federal do Piauí, Brasil

E-mail: laildasantos@hotmail.com

Sandra Valéria Nunes Barbosa

ORCID: https://orcid.org/0000-0003-4281-8807

Universidade Federal do Piauí, Brasil

E-mail: sandranunesb79@gmail.com

Luciane Resende da Silva Leonel

ORCID: https://orcid.org/0000-0003-1787-1673

Universidade Federal do Piauí, Brasil

E-mail: lucianeresendelinda@gmail.com

\section{Resumo}

Para melhorar os serviços de saúde com foco na qualidade e segurança, faz-se necessário que ocorram mudanças concretas, profundas, práticas e relativamente simples. Essas transformações são possíveis, e uma das estratégias que pode ser utilizada é a metodologia lean. O estudo objetivou analisar o impacto da metodologia Lean para a seguraça do paciente hospitalizado. A pesquisa é de caráter descritiva, do tipo revisão integrativa da literatura. A busca foi realizada entre os meses de setembro a novembro de 2019, mediante consulta nas bases de dados: MEDLINE/PubMed, LILACS, BDENF E IBECS. Os resultados após análise foram agrupados em duas categorias: 1) Uso da metodologia Lean para melhorar os processos de gestão nos serviços de saúde; 2) A metodologia Lean como 
ferramenta para aprimorar os processos de cuidado e a segurança do paciente. Diante dos estudos analisados foi evidenciado que a metodologia Lean impacta na qualidade da assistência, demonstra o quanto uma organização enxuta pode favorecer melhorias nos processos de gestão, assistenciais e clínicos, e consequentemente a segurança do paciente.

Palavras-chave: Lean; Segurança do paciente; Hospital; Paciente internado.

\begin{abstract}
To improve healthcare services with a focus on quality and safety, it is necessary that concrete, profound, practical, and relatively simple changes occur. These transformations are possible, and one of the strategies that can be used is the lean methodology. The study aimed to analyze the impact of the Lean methodology for the safety of hospitalized patients. The research is descriptive, of the integrative literature review type. The search was conducted between September and November 2019, by consulting the databases: MEDLINE/PubMed, LILACS, BDENF and IBECS. The results after analysis were grouped into two categories: 1) Use of Lean methodology to improve management processes in health services; 2) Lean methodology as a tool to improve care processes and patient safety. The studies analyzed showed that the Lean methodology impacts the quality of care, demonstrating how a lean organization can promote improvements in management, care and clinical processes, and consequently patient safety.
\end{abstract}

Keywords: Lean; Patient safety; Hospital; Inpatient.

\title{
Resumen
}

Para mejorar los servicios sanitarios centrándose en la calidad y la seguridad, es necesario que se produzcan cambios concretos, profundos, prácticos y relativamente sencillos. Estas transformaciones son posibles, y una de las estrategias que se pueden utilizar es la metodología lean. El estudio pretendía analizar el impacto de la metodología Lean en la seguridad del paciente hospitalizado. La investigación es descriptiva, del tipo revisión bibliográfica integradora. La búsqueda se realizó entre septiembre y noviembre de 2019, consultando las bases de datos: MEDLINE/PubMed, LILACS, BDENF y IBECS. Los resultados tras el análisis se agruparon en dos categorías: 1) Uso de la metodología Lean para mejorar los procesos de gestión en los servicios sanitarios; 2) Metodología Lean como herramienta para mejorar los procesos asistenciales y la seguridad de los pacientes. Los estudios analizados demostraron que la metodología Lean repercute en la calidad de la atención, demostrando cómo una organización Lean puede promover mejoras en la gestión, la atención y los procesos clínicos y, en consecuencia, la seguridad del paciente.

Palabras clave: Lean; Seguridad de los pacientes; Hospital; En régimen de internado.

\section{Introdução}

O movimento de segurança do paciente ocorreu após a publicação de um livro-relatório pelo Institute of Medicine dos EUA, no ano de 1999, intitulado: "Errar é Humano - Construindo um Sistema de Saúde mais Seguro". O relatório mostrou que cerca de 100 mil pessoas morreram em hospitais anualmente vítimas de eventos adversos nos EUA, ficando como oitava causa de morte, além do grande impacto financeiro (Brasil, 2014).

$\mathrm{Na}$ publicação foram destacadas quatro afirmações principais: o problema dos danos causados por problemas adversos é grave; o principal problema está em sistemas falhos e não em falhas de pessoas; é necessário redesenhar os sistemas; e a segurança do paciente deve se tornar uma prioridade (Brasil, 2013).

Estudos realizados em outros países que utilizaram o mesmo método do estudo do Instituto Americano, confirmaram uma alta incidência de Eventos Adversos. Em média, 10\% dos pacientes internados sofrem algum tipo de evento adverso e destes 50\% são evitáveis (Mendes, 2013).

Incidentes associados ao cuidado de saúde, e em particular os eventos adversos (incidentes com danos ao paciente), representam uma elevada morbidade e mortalidade nos sistemas de saúde de todo o mundo. Em atenção à Segurança Mundial ao Paciente, em 2004, a Organização das Nações Unidas (ONU) criou a World Alliance for Patient Safety que passou a se chamar de Patient Safety Program, na qual tinha como principais objetivos organizar os conceitos e as definições sobre segurança do paciente e propor medidas para reduzir os riscos e diminuir os eventos adversos (Brasil, 2014).

Considerando a prioridade dada a Segurança do Paciente em serviços de saúde e ainda, que a gestão de riscos voltada para a qualidade e segurança do paciente englobam princípios e diretrizes, foi instituído o Programa Nacional de Segurança do Paciente no Brasil, por meio da Portaria $n^{\circ} 529$, de $1^{\text {o }}$ de abril de 2013. A Portaria $n^{\circ} 529$ tem como objetivo geral contribuir para a qualificação do cuidado em saúde em todos os estabelecimentos de saúde do território nacional (Brasil, 2013). 
Atualmente diversos incentivos e estudos direcionados a segurança do paciente estão nos Serviços de Saúde do Brasil, reforçam a importância de implementação de uma cultura de segurança do paciente nos serviços de saúde, assim como a efetivação e implementação de protocolos direcionados a este tema, para isso pode-se utilizar várias estratégias que se adequem melhor a realidade da instituição (Brasil, 2013).

Os gestores podem medir, avaliar e controlar os resultados do processo de trabalho, por meio de indicadores de desempenho, essas informações permitem chegar a resultados que medem a eficiência institucional. Os dados para o desenvolvimento das informações necessitam ser confiáveis, e por meio destas devem ser elaboradas ações que visem a melhores resultados no serviço (Negri \& Campos, 2015).

A dinâmica do processo de trabalho é muito diferente nos diversos tipos de serviços de saúde, no entanto, para atender as necessidades, as expectativas dos clientes e atingir a excelência, é essencial que os gestores das organizações construam e pratiquem uma política de qualidade, atrelada a um contínuo monitoramento, o que viabiliza produtos e serviços com maior uniformidade, com redução de não conformidades, menores custos, evitando o desperdício e o retrabalho (Miguel, 2001).

Para melhorar os serviços de saúde com foco na qualidade e segurança, faz-se necessário que ocorram mudanças concretas, profundas, práticas e relativamente simples. Essas transformações são possíveis, e uma das estratégias que pode ser utilizada é a Metodologia Lean. A aplicação do pensamento lean é uma alternativa inovadora para enxergar e resolver problemas através de suas causas profundas. O sistema de saúde precisa melhorar seus processos e com isso diminuir erros, custos e desperdícios, para que possam entender as reais necessidades dos seus clientes e assim entregar o que desejam (Pinto \& Battaglia, 2014).

O pensamento lean é uma forma diferente de raciocínio que se baseia no local de trabalho, onde se aprende a encontrar e enfrentar os problemas a serem resolvidos a partir das experiências diárias de trabalho, envolvendo toda a equipe e experimentos práticos controlados, buscando a melhoria contínua (Ballé, 2019). Esse modelo de produção enxuta foi criado pela Toyota que desenvolveu o Sistema de Produção da Toyota (Toyota Production System- TPS), essa estratégia busca melhorar a produtividade, reduzir custo (Maia, Alves \& Leão, 2011) e utiliza ferramentas para atingir seu objetivo. A adaptação do lean nos serviços de saúde tem evoluído e se tornado cada vez mais evidente como estratégia organizacional.

Diante do exposto e visando conhecer as estratégias utilizadas, por meio do sistema lean, como metodologia para melhorar a segurança do paciente nos hospitais, através do monitoramento e organização de atividades a serem desenvolvidas de forma contínua em busca de uma assistência mais segura, foi que surgiu o questionamento: Qual o impacto da metodologia lean para a segurança do paciente hospitalizado?

Dessa forma objetiva-se: Analisar o impacto da metodologia lean para a segurança do paciente hospitalizado.

\section{Método}

O presente estudo é de caráter descritivo, do tipo revisão integrativa da literatura, onde se busca o conhecimento sobre todas as informações relevantes a respeito da temática estudada. Sendo conduzida em seis etapas: 1) definição da questão de pesquisa; 2) amostragem ou busca na literatura; 3) extração dos dados dos estudos incluídos; 4) avaliação das produções; 5) interpretação dos resultados; e 6) síntese do conhecimento ou apresentação da revisão (Mendes, 2019).

Para a elaboração da questão norteadora, utilizou-se a estratégia PICO, definindo-se: P=população: "Pacientes hospitalizados"; I=interesse: Lean; C= comparação: sem grupo comparação e $\mathrm{O}=$ outcomes = desfecho: "segurança do paciente" (Melnyk, 2011). Assim, a questão deste estudo foi: Qual o impacto da metodologia lean na segurança do paciente hospitalizado? 
Foram incluídos estudos de fonte primária, publicados em qualquer idioma, sem recorte temporal, e que abordassem sobre o uso da metodologia lean em hospitais visando a segurança do paciente. Definiram-se como critérios de exclusão dissertações, teses, editoriais e artigos duplicados nas bases de dados.

A busca foi realizada entre os meses de setembro de 2019 a novembro de 2019 , mediante consulta nas seguintes bases de dados: MEDLINE/PubMed, LILACS, BDENF E IBECS VIA BVS. Os descritores foram selecionados por meio de consulta aos termos do Medical Subject Headings (MeSH) e Descritores em Ciências da Saúde (DeCS), conforme Quadro 1.

Quadro 1 - Descritores controlados e não controlados utilizados para recuperação dos artigos nas bases de dados. Teresina, PI, Brasil, 2019.

\begin{tabular}{|c|c|c|c|}
\hline & PICO & Descritores Controlados & Descritores não controlado \\
\hline \multirow[t]{4}{*}{ MESH } & $\mathbf{P}$ & Hospitals; Patients; Inpatients & Hospital; Patient; Clients; Client;Inpatient \\
\hline & $\mathbf{I}$ & - & Lean thinking; healthcar \\
\hline & $\mathbf{C}$ & - & - \\
\hline & $\mathbf{O}$ & Patient Safety; Safety Management & $\begin{array}{l}\text { Patient Safeties; Safeties, Patient; Safety, Patient; } \\
\text { Management, Safety; Safety Culture; Culture, Safety; } \\
\text { Cultures, Safety; } \\
\text { Safety Cultures; Surveillance; Surveillance }\end{array}$ \\
\hline \multirow[t]{4}{*}{ DECS } & $\mathbf{P}$ & Hospitais; Pacientes; Pacientes Internados & $\begin{array}{l}\text { Hospitales; Hospitals; Centro Hospitalar; Centros } \\
\text { Hospitalares; Hospital; Nosocômio; Nosocômios; } \\
\text { Patients; Pacientes; Clientes; Doente; Doentes; } \\
\text { Enferma; Enfermo; Paciente; Inpatients; Pacientes } \\
\text { Internos }\end{array}$ \\
\hline & I & - & $\begin{array}{l}\text { Lean thinking; mentalidade enxuta; pensamento enxuto; } \\
\text { pensamento Lean; Lean; Lean healthcar; Lean na saúde }\end{array}$ \\
\hline & $\mathbf{C}$ & - & - \\
\hline & $\mathbf{O}$ & $\begin{array}{lllll}\text { Segurança } & \text { do } & \text { Paciente; } & \text { Gestão da } \\
\text { Segurança } & & & & \end{array}$ & $\begin{array}{l}\text { Patient Safety; Seguridad del Paciente; Safety } \\
\text { Management; Administración de la Seguridad; } \\
\text { Gerenciamento de Segurança; Gestão de Segurança; } \\
\text { Gestão do Perigo; Monitoramento de Riscos e Efeitos } \\
\text { Adversos }\end{array}$ \\
\hline
\end{tabular}

Fonte: Autores (2019).

A combinação dos descritores possibilitou a construção da estratégia de busca, que foi adaptada de acordo com as especificidades de acesso a cada base, tendo como eixos norteadores a pergunta de pesquisa e os critérios de inclusão previamente definidos.

O Quadro 2 apresenta a estratégia de busca realizada na PubMed e o Quadro 3 a busca realizada na BVS. 
Quadro 2 - Estratégia de busca realizada na base de dados PubMed. Teresina, PI, Brasil, 2019.

\begin{tabular}{|c|c|}
\hline & Estratégia de busca \\
\hline $\mathbf{P}$ & $\begin{array}{l}\text { (("inpatients"[MeSH Terms] OR "inpatients"[All Fields]) OR ("inpatients"[MeSH Terms] OR "inpatients"[All Fields] } \\
\text { OR "inpatient"[All Fields])) OR ((((("patients"[MeSH Terms] OR "patients"[All Fields]) OR ("patients"[MeSH } \\
\text { Terms] OR "patients"[All Fields] OR "patient"[All Fields])) OR ("patients"[MeSH Terms] OR "patients"[All Fields] } \\
\text { OR "clients"[All Fields])) OR ("patients"[MeSH Terms] OR "patients"[All Fields] OR "client"[All Fields])) AND } \\
((\text { "hospitals"[MeSH Terms] OR "hospitals"[All Fields]) OR ("hospitals"[MeSH Terms] OR "hospitals"[All Fields] OR } \\
\text { "hospital"[All Fields]))) }\end{array}$ \\
\hline I & $\begin{array}{l}\text { ((Lean[All Fields] AND ("thinking"[MeSH Terms] OR "thinking"[All Fields])) OR Lean[All Fields]) OR (lean[All } \\
\text { Fields] AND ("delivery of health care"[MeSH Terms] OR ("delivery"[All Fields] AND "health"[All Fields] AND } \\
\text { "care"[All Fields]) OR "delivery of health care"[All Fields] OR "healthcare"[All Fields])) }\end{array}$ \\
\hline $\mathbf{C}$ & - \\
\hline $\mathbf{O}$ & 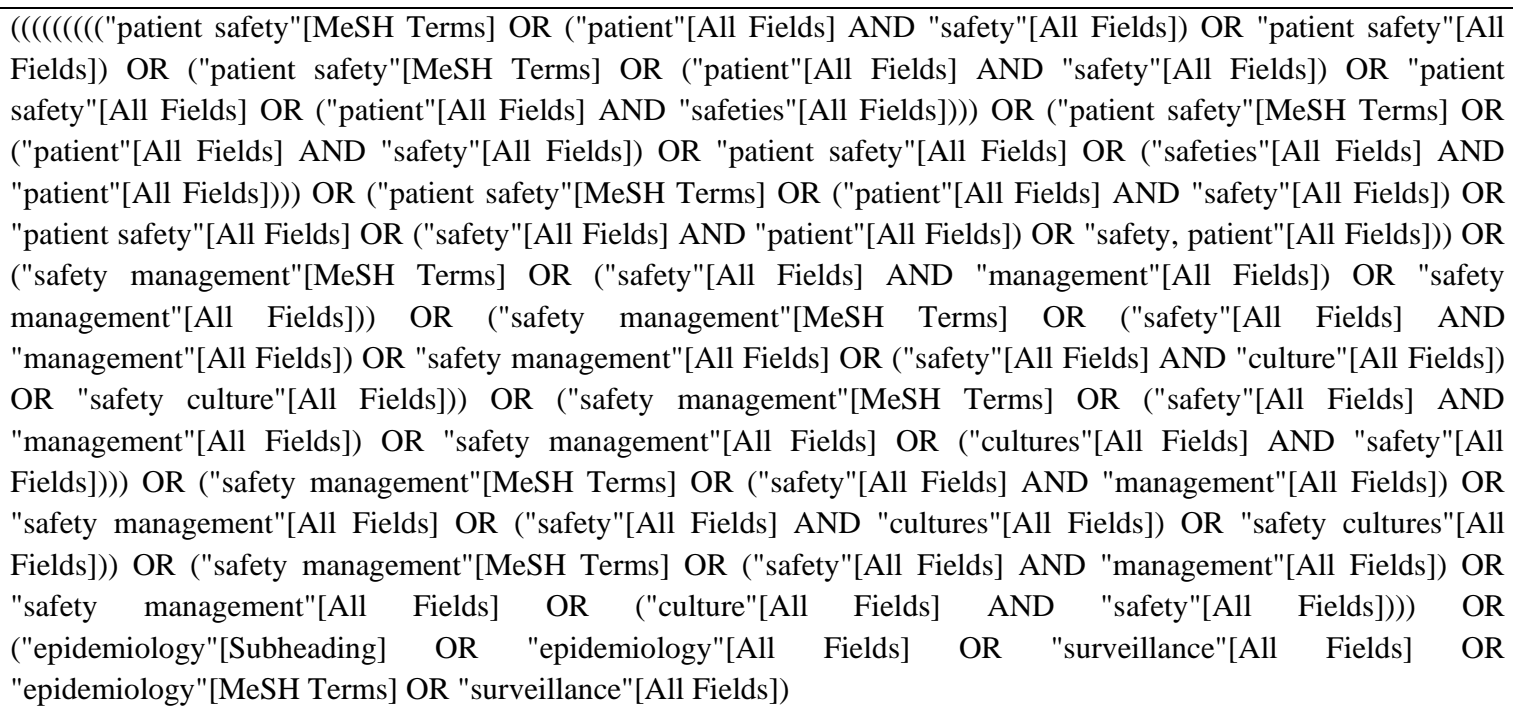 \\
\hline & P AND I AND O \\
\hline & 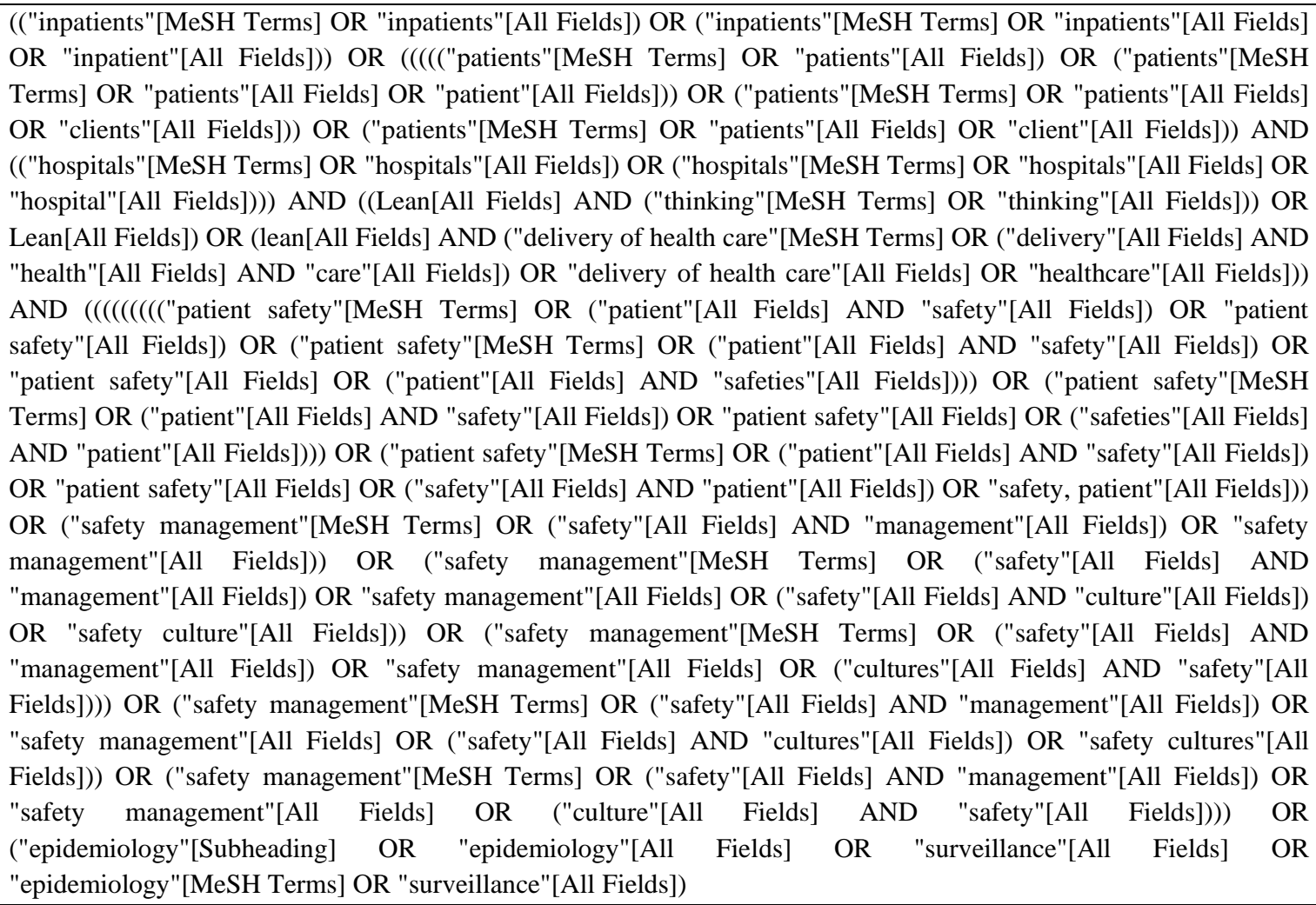 \\
\hline
\end{tabular}

Fonte: Autores (2019). 
Quadro 3 - Estratégia de busca realizada na base de dados LILACS, BDENF e IBECS VIA BVS. Teresina, PI, Brasil, 2019.

\begin{tabular}{|l|l|l|}
\hline & \multicolumn{1}{|c|}{ Estratégia de busca } \\
\hline $\mathbf{P}$ & $(((\mathrm{mh}:($ Pacientes)) OR (tw:(Pacientes)) OR (tw:(Patients )) OR (tw:(Clientes)) OR (tw:(Doente)) OR (tw:(Doentes)) \\
& OR (tw:(Enferma)) OR (tw:(Enfermo)) OR (tw:(Paciente))) AND ((mh:(Hospitais)) OR (tw:(Hospitais)) OR \\
& $\begin{array}{l}\text { (tw:(Hospitales)) OR (tw:(Hospitals)) OR (tw:("Centro Hospitalar")) OR (tw:("Centros Hospitalares")) OR } \\
\text { (tw:(Hospital)) OR (tw:(Nosocômio)) OR (tw:(Nosocômios ))) OR ((mh:("Pacientes Internados")) OR }\end{array}$ \\
\hline I & (tw:("Pacientes Internados")) OR (tw:(Inpatients)) OR (tw:("Pacientes Internos"))) \\
\hline C & (tw:("Lean thinking")) OR (tw:("mentalidade enxuta")) OR (tw:("pensamento enxuto")) OR (tw:("pensamento Lean")) \\
\hline OR (tw:(Lean)) OR (tw:("Lean healthcar")) OR (tw:("Lean na saúde"))
\end{tabular}

Fonte: Autores (2019).

As produções foram acessadas por meio do portal de periódicos da Coordenação de Aperfeiçoamento de Pessoal de Nível Superior (CAPES), sendo a busca e a seleção realizadas de forma independente por dois revisores, que, após leitura de títulos, resumos e inclusão dos estudos, obtiveram índice de concordância superior a 70\% (Whittemore \& Knafl, 2005).

Inicialmente, foram localizadas 686 produções, das quais 76, tinham relação com o tema, desses 21 atenderam aos critérios de inclusão, sendo selecionadas para o estudo. Depois da leitura do texto integral, 08 foram excluídos por duplicidade nas bases de dados, resultando em uma amostra de 13 artigos. A Figura 1 descreve o percurso realizado para a identificação, a inclusão e a exclusão dos estudos, segundo a base consultada. 
Figura 1 - Percurso para recuperação e seleção dos estudos nas bases de dados investigadas. Teresina, PI, Brasil, 2019

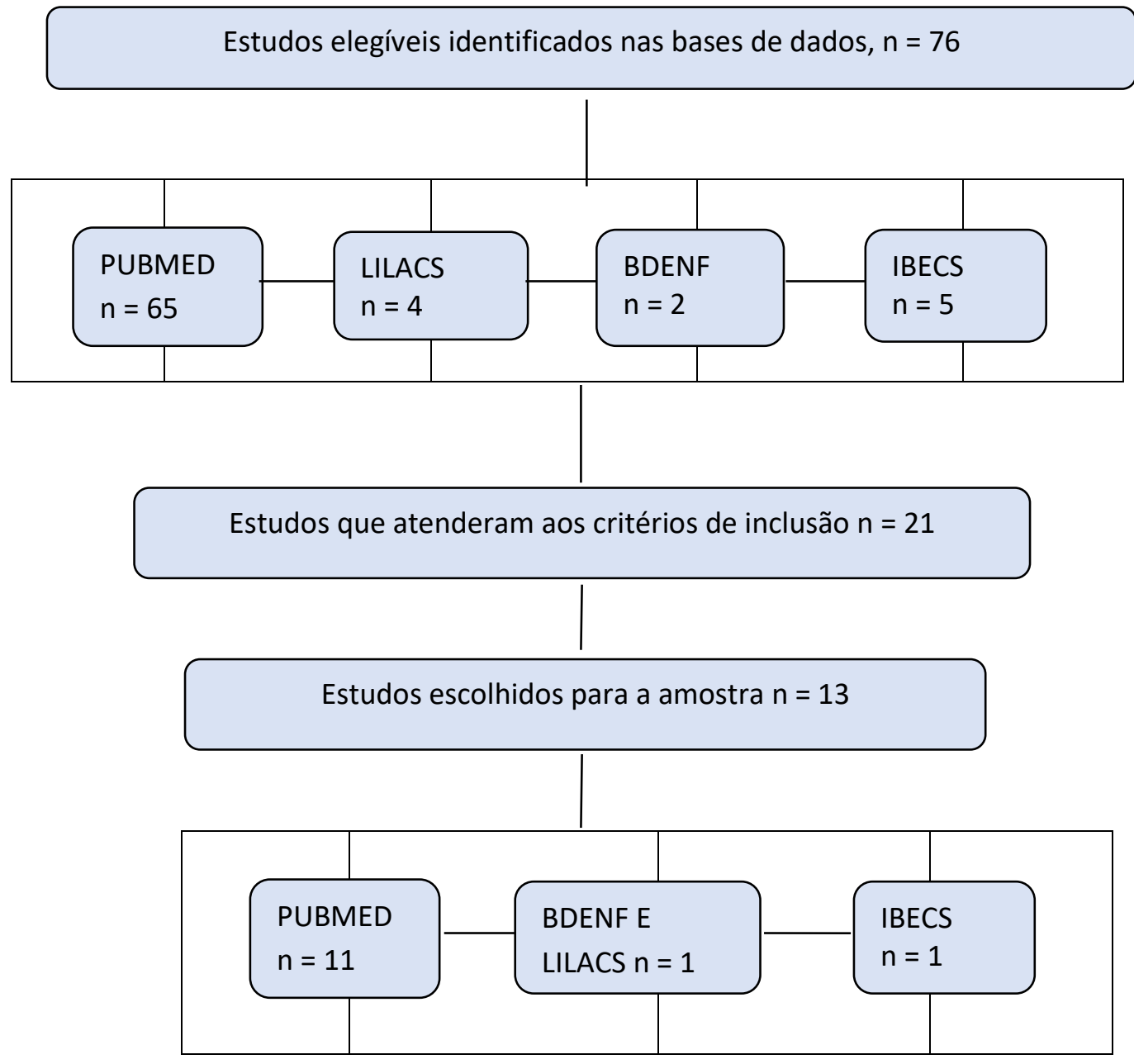

Fonte: Autores (2019).

A extração dos dados foi realizada com auxílio de instrumento próprio, contendo informações sobre título do artigo, autor (es), periódico, ano de publicação, delineamento da pesquisa, principais resultados dos estudos, nível de evidência (NE) e grau de recomendação proposto por Atallah (1998).

Para a análise do Nível de Evidência (NE), adotou-se os conceitos do sistema GRADE (Brasil, 2014), os quais consideram: a numeração caracteriza a recomendação, onde 1 é forte e 2 é fraca, e as letras mostram a qualidade da evidencia, onde A é alta; B moderada; C baixa e D muito baixa.

A análise e síntese dos dados foram realizadas de forma descritiva, e as produções selecionadas foram organizadas em planilhas, procedendo-se com a construção de quadros de acordo com as variáveis identificadas. Foi realizado o ordenamento do material e a classificação por similaridade semântica, o que possibilitou a construção de duas (02) categorias temáticas.

\section{Resultados e Discussão}

Os resultados são apresentados no Quadro 4, em conformidade com referência, autor principal, periódico, ano de publicação, tipo de estudo, objetivos, principais resultados e NE.

O ano com maior número de artigos publicados foi 2013, com três estudos, seguido de 2017, 2018 e 2019 , cada um com dois estudos. Ressalta-se que o estudo mais antigo que aborda a temática data de 2007, e o mais recente, de 2019. 
Quanto ao idioma, onze artigos foram publicados em inglês, um em espanhol e um em português. Das bases de dados pesquisadas onze foram identificados na MEDLINE/PubMed, um na LILACS e BDENF e um na IBECS. No que concerne ao delineamento dos estudos, dois são transversais e onze qualitativos. Destes últimos, seis são de intervenção, um estudo de coorte, um estudo prospectivo randomizado, uma entrevista com especialistas, um estudo de caso e um quase-experimental classificados.

Os resultados foram agrupados nas seguintes categorias: 1) Uso da metodologia lean para melhorar os processos de gestão nos serviços de saúde; 2) A metodologia lean como ferramenta para aprimorar os processos de cuidado e a segurança do paciente. Os estudos apresentados no Quadro 4 abordam sobre o uso da filosofia lean em diversos cenários.

Quadro 4 - artigos identificados com especificação individual por categorias - Teresina, PI, Brasil, 2019.

\begin{tabular}{|c|c|c|c|c|}
\hline $\begin{array}{l}\text { Autor principal, } \\
\text { periodico e ano }\end{array}$ & Delineamento & Objetivo & Fatores & $\mathbf{N E}$ \\
\hline $\begin{array}{l}\text { Ahmed, S./ Int J } \\
\text { Health Care Qual / } \\
2018\end{array}$ & Estudo Transversal & $\begin{array}{l}\text { Investigar os efeitos do Lean } \\
\text { Six Sigma (LSS) e } \\
\text { gerenciamento da força de } \\
\text { trabalho no desempenho da } \\
\text { qualidade dos hospitais da } \\
\text { Malásia. }\end{array}$ & $\begin{array}{l}\text { A abordagem Lean Six Sigma } \\
\text { melhora a força de trabalho. A } \\
\text { gestão tem influência direta e } \\
\text { significativa no desempenho da } \\
\text { qualidade nos hospitais da } \\
\text { Malásia. } \\
\text { A gestão não tem impacto de } \\
\text { forma direta, indiretamente seu } \\
\text { impacto é significativo no } \\
\text { desempenho da qualidade. }\end{array}$ & $1 \mathrm{C}$ \\
\hline $\begin{array}{l}\text { Allaudeen, N./ Qual } \\
\text { Manag Health Care } \\
\text { / } 2017\end{array}$ & 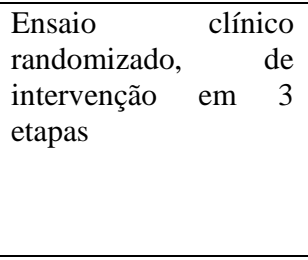 & $\begin{array}{l}\text { Avaliar uma iniciativa baseada } \\
\text { no lean para reduzir a ED LOS } \\
\text { para admissões de } \\
\text { medicamentos }\end{array}$ & $\begin{array}{l}\text { O gerenciamento lean reduziu } \\
\text { significativamente ED (duração } \\
\text { de estadia) LOS (tempo de } \\
\text { permanência (LOS) } \\
\text { para admissão de medicamentos. } \\
\text { E sustentou esses resultados ao } \\
\text { longo de vários anos. }\end{array}$ & $1 \mathrm{~B}$ \\
\hline $\begin{array}{l}\text { Blijleven, V/ Int J } \\
\text { Med Inform / } 2017\end{array}$ & $\begin{array}{l}\text { Abordagem } \\
\text { qualitativa com } \\
\text { observações diretas e } \\
\text { entrevistas } \\
\text { semiestruturadas. }\end{array}$ & $\begin{array}{l}\text { avaliar o impacto de um sistema } \\
\text { eletrônico de registros de saúde } \\
\text { de um grande hospital } \\
\text { universitário nos fluxos de } \\
\text { trabalho dos profissionais de } \\
\text { saúde, sob uma perspectiva de } \\
\text { gerenciamento de resíduos } \\
\text { enxutos. }\end{array}$ & $\begin{array}{l}\text { A perspectiva de resíduos } \\
\text { enxutos pode informar o (re) } \\
\text { design de sistemas de RSE e } \\
\text { eliminar ineficiências, } \\
\text { contribuindo para aumentar a } \\
\text { segurança, eficácia e eficiência } \\
\text { do paciente de cuidados }\end{array}$ & $1 \mathrm{~B}$ \\
\hline $\begin{array}{l}\text { Creed, M./ J Nurs } \\
\text { Care Qual/2019 }\end{array}$ & $\begin{array}{l}\text { Intervenção } \\
\text { multifacetada }\end{array}$ & $\begin{array}{l}\text { Reduzir em } 25 \% \text { as viagens dos } \\
\text { enfermeiros à farmácia para } \\
\text { liberar o tempo de enfermagem }\end{array}$ & $\begin{array}{l}\text { Redução nas jornadas de } \\
\text { enfermagem para a farmácia } \\
\text { para buscar medicamentos } \\
\text { controlados e tempo da farmácia }\end{array}$ & $1 \mathrm{C}$ \\
\hline $\begin{array}{l}\text { Ching, Joan M/ The } \\
\text { Joint Commission } \\
\text { Journal on Quality } \\
\text { and Patient } \\
\text { Safety/2013 }\end{array}$ & $\begin{array}{l}\text { Estudo de coorte } \\
\text { Baseado } \\
\text { prospectivamente por } \\
21 \text { meses, usando o } \\
\text { CALNOC (Métodos } \\
\text { de estudo da } \\
\text { qualidade da precisão) }\end{array}$ & $\begin{array}{l}\text { Identificar e eliminar } \\
\text { sistematicamente o desperdício } \\
\text { e a ineficiência em todos os } \\
\text { processos que fazem parte da } \\
\text { prestação de cuidados de saúde }\end{array}$ & $\begin{array}{l}\text { Nossos resultados sugerem que } \\
\text { o estudo CALNOC apóia a } \\
\text { princípio lean fundamental da } \\
\text { inspeção no local de trabalho e } \\
\text { que esforços rigorosos de QI } \\
\text { Lean podem contribuir para } \\
\text { diminuir a muitos ferimentos e } \\
\text { mortes que ocorrem a cada ano } \\
\text { devido a medicamentos. }\end{array}$ & $1 \mathrm{~B}$ \\
\hline $\begin{array}{l}\text { Costa, Diovane } \\
\text { Ghignatti da / Esc } \\
\text { Anna Nery/ } 2018\end{array}$ & $\begin{array}{l}\text { Estudo de caso, do } \\
\text { tipo exploratório e } \\
\text { descritivo }\end{array}$ & $\begin{array}{l}\text { analisar a situação atual e } \\
\text { aplicar um método para } \\
\text { estabelecer prioridades entre os } \\
\text { problemas levantados para o } \\
\text { planejamento de melhorias, } \\
\text { com base no pensamento Lean. }\end{array}$ & $\begin{array}{l}\text { Identificação sistemática de } \\
\text { atividades que agregam valor ou } \\
\text { que geram perdas no preparo e } \\
\text { administração de medicamentos, } \\
\text { com vistas à melhoria efetiva do } \\
\text { processo de medicação com o } \\
\text { envolvimento da equipe. }\end{array}$ & $1 \mathrm{D}$ \\
\hline $\begin{array}{l}\text { Font Noguera A, / } \\
\text { Revista de Calidad } \\
\text { Asistencial/ } 2013\end{array}$ & $\begin{array}{l}\text { Estudo observacional } \\
\text { e prospectivo }\end{array}$ & $\begin{array}{l}\text { Analisar os resultados obtidos } \\
\text { com a metodologia Lean Six } \\
\text { Sigma no diagnóstico e a }\end{array}$ & $\begin{array}{l}\text { A metodologia Lean Six Sigma } \\
\text { tem sido uma estratégia de } \\
\text { melhoria contínua. }\end{array}$ & $1 \mathrm{C}$ \\
\hline
\end{tabular}




\begin{tabular}{|c|c|c|c|c|}
\hline & & $\begin{array}{lrr}\text { melhoria } & \text { do } & \text { processo } \\
\text { farmacoterapêutico do paciente } & \\
\text { hospitalizado } & \text { durante } & \text { a } \\
\text { mudança } & \text { estrutural } & \text { e } \\
\text { organização de um hospital } \\
\text { terciário. }\end{array}$ & & \\
\hline $\begin{array}{l}\text { Furman, C./ Jt } \\
\text { Comm J Qual } \\
\text { Patient Saf / } 2007\end{array}$ & Intervenção & $\begin{array}{l}\text { Traduzir as técnicas de zero } \\
\text { defeitos e parar a linha em } \\
\text { assistência médica, o sistema } \\
\text { Alerta de Segurança do } \\
\text { Paciente (PSA) }\end{array}$ & $\begin{array}{lcc}\text { Melhoria da cultura } & \text { de } \\
\text { segurança. } & \text { Melhorias } & \text { da } \\
\text { segurança e } & \text { qualidade. } & \end{array}$ & $1 \mathrm{C}$ \\
\hline $\begin{array}{l}\text { Huhs, E./ Evid } \\
\text { Fortbild Qual } \\
\text { Gesundhwes/ } 2019\end{array}$ & $\begin{array}{lr}\text { Abordagem } & \text { semi- } \\
\text { qualitativa, } & \text { com } \\
\text { entrevistas } & \text { com } \\
\text { especialistas. } & \end{array}$ & $\begin{array}{l}\text { usar entrevistas com } \\
\text { especialistas do setor de saúde } \\
\text { para descobrir quais hospitais } \\
\text { de filosofia de liderança } \\
\text { precisam para implementar com } \\
\text { sucesso a abordagem de } \\
\text { gerenciamento enxuto em sua } \\
\text { cultura organizacional. }\end{array}$ & $\begin{array}{l}\text { A metodologia lean melhora a } \\
\text { qualidade e segurança. Gestores } \\
\text { tem dificuldades de implantar o } \\
\text { Lean de forma generalizada. } \\
\text { Exige engajamento do gestor e } \\
\text { de toda a equipe }\end{array}$ & 1D \\
\hline $\begin{array}{l}\text { Hintzen, B. L. / Am } \\
\text { J Health Syst } \\
\text { Pharm } \\
\text { /2009 }\end{array}$ & Intervenção & $\begin{array}{l}\text { Implementar técnicas enxutas } \\
\text { na farmácia de um hospital para } \\
\text { melhorar o fluxo de trabalho, } \\
\text { reduzir desperdício e obter } \\
\text { economias substanciais. }\end{array}$ & $\begin{array}{l}\text { Benefícios deste processo } \\
\text { incluiu uma economia } \\
\text { financeira, à redução de } \\
\text { resíduos, melhorias no fluxo de } \\
\text { trabalho e diminuição requisitos } \\
\text { de pessoal. }\end{array}$ & $1 \mathrm{C}$ \\
\hline
\end{tabular}

Categoria 2: A metodologia lean como ferramenta para aprimorar os processos de cuidado e a segurança do paciente

\begin{tabular}{|c|c|c|c|c|}
\hline $\begin{array}{l}\text { Blackmore, E. / Jt } \\
\text { Comm J Qual } \\
\text { Patient Saf/ } 2013\end{array}$ & $\begin{array}{l}\text { Estudo prospectivo } \\
\text { quase-experiemental. }\end{array}$ & $\begin{array}{l}\text { avaliar a eficácia das } \\
\text { intervenções Lean QI em } \\
\text { diminuindo a taxa de erro no } \\
\text { processamento de instrumentos } \\
\text { cirúrgicos e quantificar a } \\
\text { frequência e o caráter dos erros } \\
\text { vistos. }\end{array}$ & $\begin{array}{l}\text { Melhoria da qualidade cirúrgica } \\
\text { (QI) e erros cirúrgicos de } \\
\text { instrumentos estéreis com } \\
\text { significativamente redução pela } \\
\text { aplicação rigorosa de } \\
\text { Metodologia VMPS Lean QI, } \\
\text { com potencial melhoria em } \\
\text { qualidade e eficiência cirúrgicas. }\end{array}$ & $1 \mathrm{~B}$ \\
\hline 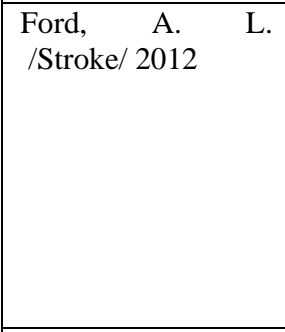 & $\begin{array}{l}\text { Estudo de intervenção } \\
\text { coorte e prospectivo } \\
\text { de comparação (antes } \\
\text { e depois) }\end{array}$ & $\begin{array}{l}\text { Uso da metodologia de } \\
\text { melhoria de processo lean para } \\
\text { desenvolver um protocolo tPA } \\
\text { intravenoso simplificado. }\end{array}$ & $\begin{array}{l}\text { Os princípios de fabricação } \\
\text { enxuta foram aplicados para } \\
\text { agilizar a aplicação do tPA } \\
\text { (ativador de plasminogênio do } \\
\text { tipo tecido) por via intravenosa } \\
\text { com redução drástica nos DNTs } \\
\text { (tempo de início da aplicação) e } \\
\text { sem comprometer a segurança } \\
\text { do paciente. }\end{array}$ & 1B \\
\hline $\begin{array}{l}\text { Johnson, A. E./ Am } \\
\text { J Med Qual/ } 2015\end{array}$ & $\begin{array}{l}\text { Este estudo } \\
\text { prospectivo, pré e } \\
\text { pós-intervenção, } \\
\text { quase-experiental }\end{array}$ & $\begin{array}{l}\text { Investigar o impacto de uma } \\
\text { estrutura que mescla Lean } \\
\text { Sigma, design thinking e Lean } \\
\text { Startup em readmissões por } 30 \\
\text { dias em todas as causas entre } \\
\text { pacientes com IC }\end{array}$ & $\begin{array}{l}\text { Diminuição significativa da taxa } \\
\text { de readmissões. O Lean Sigma } \\
\text { forneceu uma abordagem } \\
\text { rigorosa; Análise da causa raiz } \\
\text { gerou uma estrutura conceitual } \\
\text { em torno de quais intervenções } \\
\text { poderiam ser formuladas; } \\
\text { Design thinking permitiu confiar } \\
\text { no poder da observação, uma } \\
\text { característica inerente } \\
\text { componente do treinamento em } \\
\text { ciências da saúde, para ampliar a } \\
\text { ideia geração e inovação; O } \\
\text { Lean Startup promoveu um } \\
\text { processo de experimentação } \\
\text { rápida e simultânea para testar } \\
\text { intervenções. Juntos, melhor } \\
\text { aproveitamento dos recursos } \\
\text { locais, desenvolvimento de } \\
\text { trabalho equaipe } \\
\text { multidisciplinar e canalização de } \\
\text { criatividade. }\end{array}$ & $1 \mathrm{~B}$ \\
\hline
\end{tabular}




\section{Uso da metodologia Lean para melhorar os processos de gestão nos serviços de saúde}

Os processos de gestão na área da saúde podem ser melhorados com o uso da filosofia Lean. Pode-se observar que os estudos utilizaram o Lean em sua forma fundamental ou em conjunto com outras metodologias que trabalham com os mesmos princípios, a exemplo o six sigma. O método Lean tem sido utilizado dentro das mais diversas estruturas e departamentos (Ahmed, 2018).

A metodologia lean melhora a qualidade e segurança. Com gerenciamento lean pode-se observar significativa redução na duração de estadia e tempo de permanência dos pacientes e consequente diminuição do uso de medicamentos, tendo sustentado esses resultados ao longo de vários anos (Allaudeen, 2017). Dentre os benefícios deste processo estão uma economia financeira, à redução de resíduos, melhorias no fluxo de trabalho e diminuição requisitos de pessoal, melhorando também, a cultura de segurança (Hintzen, 2009). A abordagem Lean Six Sigma melhora a força de trabalho e tem sido uma estratégia de melhoria contínua (Ford, 2013).

Com o uso do Lean ou do Lean conjugado a outras abordagens, pode ser percebido melhorias nos mais diferentes aspectos. Relacionados a medicamentos, houveram melhorias da qualidade e esta pode contribuir para diminuir a eventos adversos e mortes que ocorrem a cada ano devido a medicamentos (Ching, 2013). No que concerne medicamentos e força de trabalho, a organização de forma enxuta levou a uma redução nas jornadas de enfermagem para a farmácia para buscar medicamentos controlados e tempo da farmácia (Creed, 2019).

O gerenciamento Lean em um serviço de saúde, reduziu significativamente o tempo de permanência para administração de medicamentos e sustentou esses resultados ao longo de vários anos. Apresentou melhoria efetiva do processo de medicação, com a identificação sistemática de atividades que agregam valor ou que geram perdas no preparo e administração de medicamentos (Costa, 2018).

Uma ferramenta que pode ser utilizada na perspectiva de resíduos enxutos são os sistemas informatizados. que pode informar o (re) design de sistemas de registro eletrônico na saúde (RSE) e eliminar ineficiências, contribuindo para aumentar a segurança, eficácia e eficiência do paciente de cuidados (Blijleven, 2017).

Um estudo mostrou que a gestão tem influência direta e significativa no desempenho da qualidade nos hospitais. A gestão não tem impacto de forma direta, indiretamente seu impacto é significativo no desempenho da qualidade. Contudo gestores tem dificuldades de estabelecer o Lean de forma generalizada em suas instituições, preferem implantar pontualmente nos focos dos problemas. Ao ser instituído o Lean exige engajamento do gestor e de toda a equipe para sua consolidação (Huhs, 2019). Levando a melhoria da cultura de segurança e que resulta na segurança e qualidade da assistência ao paciente.

Contabilizaram-se dez artigos nesta categoria, desses oito foram publicados no idioma inglês, um em português e um em espanhol, com níveis de evidência (NE) entre B e D e uma forte recomendação. Os estudos confirmam a importãncia de estratégia de gestão mais enxutas e direcionadas que favoreçam o direcionamento das equipes por meio de forma participativa com vista a uma cultura votada para a qualidade assistencial.

Os estudos corroboram com outros autores ao evidenciarem que a metodologia Lean, por meio do uso de ferramentas e abordagens organizacionais, propicia reduzir desperdício, melhorar a qualidade, reduzir custos e garantir um estreito alinhamento dos processos operacionais aos objetivos organizacionais (Schweikhart \& Dembe, 2009).

\section{A metodologia Lean como ferramenta para aprimorar os processos de cuidado e a segurança do paciente}

A lean como ferramenta para aprimorar os processos de cuidado e consequentemente a qualidade e segurança do paciente, pode ser percebida em todos os estudos selecionados nesta categoria. Os estudos demonstraram uma diversidade de opções em que a lean e suas ramificações, podem ser utilizados, através de suas ferramentas, e todas com resultados positivos. 
Em um estudo pode ser observado que ocorreu melhoria da qualidade cirúrgica e diminuição de erros cirúrgicos de instrumentos estéreis com significativamente redução pela aplicação rigorosa de metodologia Lean, com potencial de melhoria em qualidade e eficiência cirúrgicas (Blackmore, 2013).

Em um estudo que buscou agilizar um procedimento e diminuir o tempo de espera do paciente foi modificado o fluxo de atendimento, utilizando os princípios de fabricação enxuta para aplicação do TPA (ativador de plasminogênio do tipo tecido) por via intravenosa, o que levou a redução drástica nos DNTS (tempo de início da aplicação) e sem comprometer a segurança do paciente (Ford, 2012).

O uso do Lean Sigma, design thinking e Lean Startup de forma mesclada foi utilizada para buscar diminuir as readmissões por insuficiência cardíaca (IC) e pode-se perceber um impacto positivo com diminuição significativa da taxa de readmissões. A junção desses métodos, levou a um melhor aproveitamento dos recursos locais, desenvolvimento de trabalho em equipe multidisciplinar e canalização de criatividade (Johnson, 2015).

Para essa categoria foram utilizados três estudos que se direcionaram para uma abordagem lean em busca melhorar os processos de cuidado, todos publicados em inglês e com NE = B e com forte recomendação.

O pensamento lean tem como princípio a contínua eliminação das atividades desnecessárias, para evitar desperdícios. Esse pensamento perpassa por todos os tipos de processos, assistenciais, de suporte e administrativos. Ao eliminar esforços desnecessários, será disponibilizado mais tempo e recursos disponíveis para as coisas que realmente importam como a segurança do paciente e a qualidade do cuidado.

\section{Conclusão}

Diante dos estudos analisados foi evidenciado que a metodologia Lean impacta na qualidade da assistência, demonstra o quanto uma organização enxuta pode favorecer melhorias nos processos de gestão, assistenciais e clínicos, e consequentemente a segurança do paciente. Trazer esse método para a área da saúde é favorável, uma vez que as instituições de saúde são organizações muito complexas e diversificadas, e esse formato favorece processos bem delineados.

Apesar de existirem estudos que mostram o uso da ferramenta como positiva, ainda faz-se necessário um número maior de estudos com maior rigor metodológico para que os gestores se sintam mais seguros em utilizar o Lean e suas ferramentas.

Como limitações para a elaboração desta revisão integrativa, cita-se a não disponibilização de alguns artigos, na sua forma completa, nas bases de dados, impedindo a leitura e a avaliação detalhada do estudo.

\section{Referências}

Ahmed, S., Manaf, N. H. A. \& Islam, R. (2018). Effect of Lean Six Sigma on Quality Performance in Malaysian Hospitals. International Journal of Health Care Quality Assurance. 31(8). https://doi.org/10.1108/IJHCQA-07-2017-0138.

Allaudeen, N., Vashi, A., Breckenridge, J. S., Haji-Sheikhi, F., Wagner, S., Posley, K. A. \& Asch, S. M. (2017). Using Lean Management to Reduce Emergency Department Length of Stay for Medicine Admissions. Quality Improvement Initiatives. 26(2):91-96. 10.1097/QMH.0000000000000132

Atallah, A. N., \& Castro, A. A. (1998). Evidências para melhores decisões clínicas. Centro Cochrane do Brasil.

Balle, M., Jones, D., Chaize, J. \& Fiume, O. (2019). A estratégia Lean: Para a criar vantagem competitiva, inovar e produzir com crescimento sustentável. Bookman: Porto Alegre.

Blackmore, C. C, Bishop, R., Luker, S. \& Williams, B. L (2013). Applying Lean Methods to Improve Quality and Safety in Surgical Sterile Instrument Processing, Performance Improvement. 39(3):99-10. 10.1016/s1553-7250(13)39014-X

Blijleven, V., Koelemeijer, K., \& Jaspers, M. (2017). Identifying and Eliminating Inefficiencies in Information System Usage: A Lean Perspective. International Journal of Medical Informatics. 107:40-47. http://dx.doi.org/10.1016/j.ijmedinf.2017.08.005

Brasil. (2013). Agência Nacional de Vigilância Sanitária- ANVISA. Resolução de Diretoria Colegiada, 25 de julho de $2013 b$. Institui ações para melhoria da segurança do paciente em serviços de saúde e dá outras providências. Brasília: Diário Oficial da União. 
Brasil. (2014). Ministério da Saúde. Diretrizes metodológicas: Sistema GRADE - Manual de graduação da qualidade da evidência e força de recomendação para tomada de decisão em saúde. Brasília: Ministério da Saúde.

Brasil. (2014). Ministério da Saúde. Documento de referência para o Programa Nacional de Segurança do Paciente. Brasília: Ministério da Saúde.

Brasil. (2013). Ministério da Sáude. Portaria $n^{o} 529$, de $1^{o}$ de abril de 2013. Institui o Programa Nacional de Segurança do Paciente (PNSP). Brasília: Diário Oficial da União.

Ching, J., Long, C., Williams, B. L. \& Blackmore, C. C. (2013). Using Lean to Improve Medication Administration Safety: In Search of the "Perfect Dose". Medication Safety. 39(5):195-204. 10.1016/s1553-7250(13)39026-6

Costa, D. G., Pasin, S. S., Magalhães, A. M. M., Moura, G. M. S. S., Rosso, C. B., \& Saurin, T. A. (2018). Análise do preparo e administração de medicamentos no contexto hospitalar com base no pensamento Lean. Escola Anna Nery. 22(4):e20170402

Creed, M., McGuirkk, M., Buckley, R., Brún, A. \& Kilduff, M. (2019). Using Lean Six Sigma to Improve Controlled Drug Processes and Release Nursing Time. Care Quality. 34(3):236-241. 10.1097/NCQ.0000000000000364.

Font, N. I., Fernández, M. M., Ferrer, A., Balasch, P. S., Edo, S. M., \& Poveda, A. J. (2013). Mejora del proceso farmacoterapéutico del paciente hospitalizado mediante la metodología Lean Seis Sigma. Revista de Calidad Asistencial. 28(6):370-380. 10.1016/j.cali.2013.04.003

Ford Andria, L., Williams, J. A., Spencer, M., McCammon, C., Khoury, N., Sampson, T. R., Panagos, P., \& Lee, J. M. (2012). Reducing Door-to-Needle Times Using Toyota's Lean Manufacturing Principles and Value Stream Analysis. Methods. Stroke. 43(12):3395-8. 10.1161/STROKEAHA.112.670687

Furman, C. (2007). System: Using a Patient Safety Alert System to Reduce Error. Methods, Tools, and Strategies. 23(1):42-45.

Hintzen, B. L (2009). Effect of lean process improvement techniques on a university hospital inpatient pharmacy. Am J Health-Syst Pharm. 66(22):2042-7. 10.2146/ajhp080540.

Huhs, W., Gliebe, W. \& Sendlhofer, G. (2019). Management der Gesundheitsversorgung / Health Care Management Qualitative Analyse zu Lean Management im Gesundheitswesen:Sichtweisen österreichischer und Schweizer Experten. Qualitative analysis of lean management in healthcare: perspectives of Austrianand. Swiss expert. 143:8-14. https://doi.org/10.1016/j.zefq.2019.05.003

Johnson, A. E., Winner L, Simmons T, Eid R. H, Sampedro A, Augustine S, Sylvester C., \& Parakh K. (2015). Technology and Manufacturing Companies to Reduce Heart Failure Readmissions. American Journal of Medical Qualit. 10.1177/1062860614562627

Maia, L. C., Alves, A. C. \& Leão, C. P. (2011). Metodologias para implementar Lean Production: Uma revisão crítica de literatura. Braga: Universidade do Minho, 2011.

Melnyk, B. M. \& Fineout-Overholt, E. (2011). Making the case for evidence-based practice and cultivating a spirit of inquiry. Lippincott Williams \& Wilkins, $3-24$.

Mendes, K. D. S., Silveira, R. C. C. P., \& Galvão, C. M. (2019). Use of the bibliographic reference manager in the selection of primary studies in integrative reviews. Texto contexto-enferm. 28:e20170204. 10.1590/1980-265x-tce-2017-0204

Mendes, W., Pavão, A. L. B., Martins, M., Moura, M. L. O. \& Travassos, C. (2013). Características de eventos adversos evitáveis em hospitais do Rio de Janeiro. Revista Associação Médica Brasileira. 59(5):421-428. http://dx.doi.org/10.1016/j.ramb.2013.03.002.

Miguel, P. A. C. (2001). Qualidade: enfoques e ferramentas. Artliber.

Negri, S. C. \& Campos, M. D. (2019). O uso da ferramenta KANBAN para o controle da permanência dos usuários SUS. www.convibra.com.br /dwp.asp?id= $5191 \& \mathrm{ev}=25$

Pinto, C. F. \& Battaglia, F. (2014). Texto adaptado da Introdução do Livro “Em Busca do Cuidado Perfeito: Aplicando Lean na Saúde”. Ed. Lean Institute Brasil, São Paulo.

Schweikhart, S., \& Dembe, A. (2009). The Applicability of Lean and Six Sigma Techniques to Clinical and Translational Research. Journal of Investigative Medicine 57(7):748-55. 10.2310/JIM.0b013e3181b91b3a

Whittemore, R., \& Knafl, K. (2005). The integrative review: updated methodology. J Adv Nurs. 52(5):546-53. 10.1111/j.1365-2648.2005.03621.x 